\title{
Review Article \\ Cellular Therapeutics for Heart Failure: Focus on Mesenchymal Stem Cells
}

\author{
Amitabh C. Pandey, ${ }^{1,2}$ Jordan J. Lancaster, ${ }^{3}$ David T. Harris, ${ }^{4}$ Steven Goldman, ${ }^{5,6}$ and \\ Elizabeth Juneman ${ }^{5,6}$
}

${ }^{1}$ Department of Medicine, University of Arizona, Tucson, AZ, USA

${ }^{2}$ Division of Cardiology, Scripps Clinic, La Jolla, CA, USA

${ }^{3}$ Department of Physiology, University of Arizona, Tucson, AZ, USA

${ }^{4}$ Department of Immunobiology, University of Arizona, Tucson, AZ, USA

${ }^{5}$ Section of Cardiology, Southern Arizona Veterans Health Affairs System, Tucson, AZ, USA

${ }^{6}$ Sarver Heart Center, University of Arizona, Tucson, AZ, USA

Correspondence should be addressed to Amitabh C. Pandey; pandey.amitabh@scrippshealth.org and Elizabeth Juneman; ejuneman@shc.arizona.edu

Received 25 May 2017; Revised 31 July 2017; Accepted 14 August 2017; Published 17 December 2017

Academic Editor: Bojan Vrtovec

Copyright (C) 2017 Amitabh C. Pandey et al. This is an open access article distributed under the Creative Commons Attribution License, which permits unrestricted use, distribution, and reproduction in any medium, provided the original work is properly cited.

\begin{abstract}
Resulting from a various etiologies, the most notable remains ischemia; heart failure (HF) manifests as the common end pathway of many cardiovascular processes and remains among the top causes for hospitalization and a major cause of morbidity and mortality worldwide. Current pharmacologic treatment for HF utilizes pharmacologic agents to control symptoms and slow further deterioration; however, on a cellular level, in a patient with progressive disease, fibrosis and cardiac remodeling can continue leading to end-stage heart failure. Cellular therapeutics have risen as the new hope for an improvement in the treatment of HF. Mesenchymal stem cells (MSCs) have gained popularity given their propensity of promoting endogenous cellular repair of a myriad of disease processes via paracrine signaling through expression of various cytokines, chemokines, and adhesion molecules resulting in activation of signal transduction pathways. While the exact mechanism remains to be completely elucidated, this remains the primary mechanism identified to date. Recently, MSCs have been incorporated as the central focus in clinical trials investigating the role how MSCs can play in the treatment of HF. In this review, we focus on the characteristics of MSCs that give them a distinct edge as cellular therapeutics and present results of clinical trials investigating MSCs in the setting of ischemic HF.
\end{abstract}

\section{Introduction}

Heart failure (HF) has become a major epidemic throughout the world. Resulting as the common end pathway for a myriad of cardiovascular disease processes, HF is the most common cause of hospital admission in patients over 65 years old, with the number of individuals having HF reaching 8 million and expected costs in the United States exceeding 40-70 billion dollars [1]. The foundation of current therapy for HF is pharmaceutical interventions. Certain subsets of patients with HF may benefit from advanced therapies including cardiac resynchronization therapy (CRT), mechanical circulatory support devices, and even transplant, which is reserved to the sickest patients. However, these measures are not without pitfalls; pharmaceutical therapies have side effects, and CRT, while advantageous, is only available to some patients [2]. Recently, there has been a push to investigate more innovative treatments for HF that aim at not only improving clinical symptoms but also improving cardiovascular pathophysiology. While pharmaceutical and device therapy can improve the pathophysiology of ischemic HF, nonischemic HF still has limited options currently. One of the leading treatments under investigation for $\mathrm{HF}$ is the use of mesenchymal stem cells (MSCs). Mesenchymal stem cells are multipotent adult stem cells that have been at the forefront of regenerative medicine research. Mesenchymal 


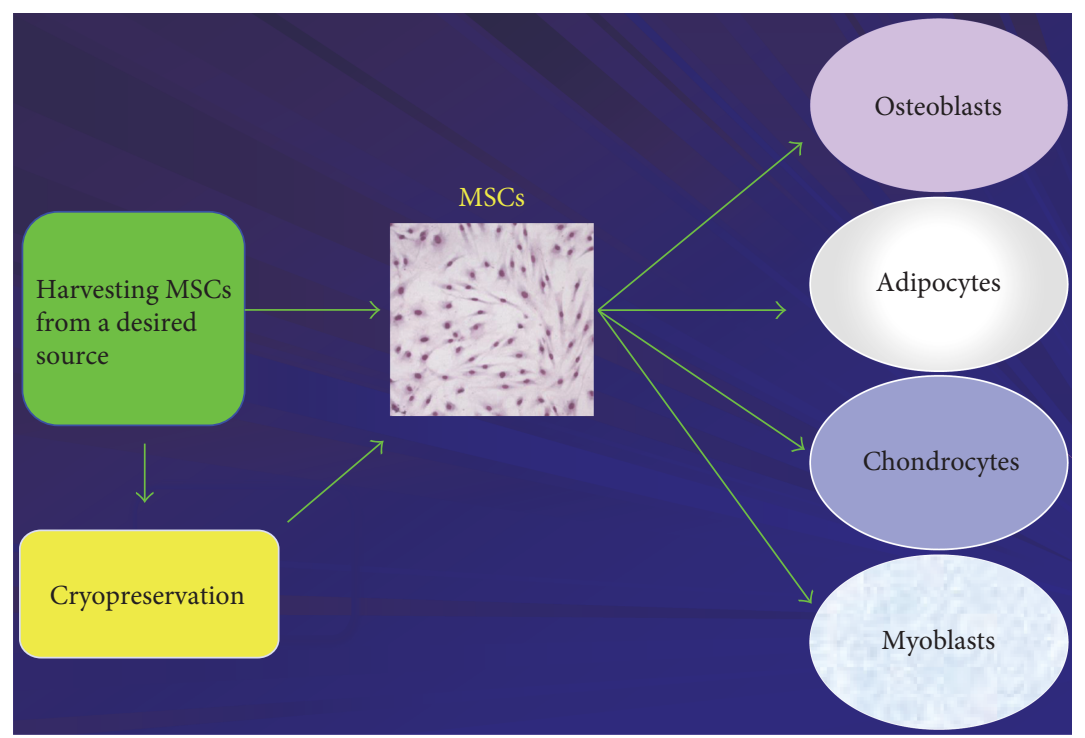

FIGURE 1: Multipotent capacity of mesenchymal stem cells. MSCs are derived from numerous tissue sources including bone marrow and adipose tissue. They are able to differentiate into various end-cell types including osteoblasts, adipocytes, chondrocytes, and myoblasts. Additionally, they are immunoprivileged, therefore allowing autologous as well as allogeneic therapeutic potential. They can also be cryopreserved, while maintaining their multipotent properties, thus allowing them to be ideal candidates for "off the shelf" cell-based therapies.

stem cells are unique cells that can be cultured ex vivo and utilized as cellular therapies in a variety of disease states. Currently, MSCs are being entertained as treatment modalities in cardiovascular disease states such as acute myocardial infarction, fibrosis, and heart failure. There are other cell therapies that have been explored in translational projects including those of induced pluripotent stem cells (iPSCs) and vector-based gene therapy. Here, we focus on MSCs and their desirable properties as cellular therapeutics in heart failure and implicate their potential use in clinical practice.

\section{Mesenchymal Stem Cells in Cell-Based Therapies}

Mesenchymal stem cells are a type of adult stem cells that are multipotent cells [3, 4]. Mesenchymal stem cells maintain the ability to give rise to a number of different end-cell lineages including bone cells, adipose cells, stromal cells, muscle cells, tendon cells, and other mesenchymal cells (Figure 1) [3-5]. Mesenchymal stem cells are utilized for endogenous cell-tocell communication and paracrine signaling and also employ these properties for cellular repair when utilized in cellular therapeutics [5]. Although not conclusively proven, mesenchymal stem cells are postulated to achieve these processes via expression of a wide spectrum of secreted factors and to a lesser extent direct end-cell differentiation for replacement of damaged cells $[4,5]$. Factors that are expressed by MSCs include cytokines, chemokines, and adhesion molecules, which then regulate the activation and/or inhibition of molecular signaling pathways for endogenous cellular repair [3]. Additionally, MSCs are immunoprivileged cells given the lack of expression of major histocompatibility complex II (MHC II) complexes in their multipotent state [6]. Furthermore, MSCs have been shown to decrease inflammation and inflammatory cues as well as to promote angiogenesis $[3,5,7]$. Mesenchymal stem cells represent an ideal candidate in the emerging field of regenerative medicine [8]. These properties combined with the accessibility of MSCs from the bone marrow or adipose tissues make MSCs ideal candidates for cell- and gene-based therapies.

Since their initiation, isolation, and description by Friedenstein and colleagues from bone marrow, MSCs have been considered potential candidates for cell-based therapies [9]. Mesenchymal stem cells have been isolated from a wide variety of tissues including bone marrow tissue, adipose tissue, cardiac tissue, umbilical cord tissue, as well as those of other sites [10-15]. The therapeutic potential of MSCs makes them the ideal candidates for cell- and gene-based therapies for a number of reasons including their applicability for "off the shelf use" potential in cellular therapeutics; their immunoprivileged state; their ability to express cytokines, chemokines, and adhesion molecules; and their ability to be expanded to sufficient therapeutic quantities ex vivo $[3,7,16]$. Previous studies have shown that MSCs maintain their therapeutic potential even after cryopreservation, further adding to the list of desirable characteristics of MSCs [17, 18]. Additionally, given their immunoprivileged state, allogeneic treatment modalities could be a legitimate possibility as well for the desired therapy in heart failure patients.

\section{MSCs and Paracrine Signaling}

While MSCs can differentiate along mesenchymal lineages to produce end-cell types needed for repopulation of damaged tissues, the primary mechanism postulated by which MSCs are able to direct and facilitate endogenous cellular repair is via paracrine signaling $[5,16]$. It is postulated that utilizing paracrine signaling, MSCs direct and modulate the cellular microenvironment by promoting survival and proliferation 
of endogenous cells, induce angiogenesis, quell inflammation, inhibit apoptosis, and recruit endogenous progenitor cells to endpoint differentiation, with an end result of functional improvement (Figure 1) [19-22]. Many studies have attempted to characterize the mechanisms by which MSCs produce a more favorable microenvironment conducive to endogenous cellular repair. It appears among the key components involved in modulating cellular signaling cascades including anti-inflammatory cues, proangiogenic signaling, and avoidance by immune surveillance [3, 4, 19, 22]. Many of the cytokines expressed by MSCs work by quelling the inflammatory signaling present in disease states including HF $[5,23,24]$. Furthermore, recent studies have shown that IL-1 $\beta$ may play a critical role in instigating the onset of HF [24]. Mesenchymal stem cells are able to secrete potent levels of interleukin 1 receptor antagonist (IL-1rn), which has been thought to be a key regulator of MSC-based therapy [5].

With MSCs available from a variety of tissues, one question that frequently arises with the proposal of MSCs as cellbased therapies is "Are MSCs taken from the varying tissues equal in directing paracrine-mediated endogenous cellular repair?" Many studies have investigated the differences in MSCs isolated from different tissue sources, and the potential of these cells remains the same despite the location of isolation [3, 25]. While MSCs possess multiple attributes desirable for the ideal cell-based therapy for HF, pitfalls do currently exist as well. Limitations as to method, timing, and dose of cells remain unknown for MSC administration in the setting of HF.

Despite the fact that MSCs can be scaled to large quantities ex vivo, limitations still exist as to delivery of an adequate number of cells given the progression and individual state of the disease for a given patient [7]. This problem seems to have been overcome with the use of allogeneic MSCs from healthy donors. Other studies have reported concerns regarding retention of injected cells over time in the heart given issues of low engraftment and limited retention of MSCs or other cell-based therapies [26]. However, given that the primary function of MSCs seems to be promotion of endogenous repair via paracrine signaling rather than direct end-cell lineage differentiation, absolute cell numbers can be deceptive. Additionally, local engraftment of cells is not entirely necessary given that paracrine signaling largely contributes to the overall function of MSCs in directing endogenous cellular repair $[27,28]$. Thus, while several mechanisms have been investigated to identify the ideal route of delivery of MSCs, further work is necessary regarding optimization of delivery to the area of injury given poor understanding of how MSCs would be best utilized [29-31]. Nonetheless, advancements have been made with recent clinical trials demonstrating safety of allogeneic MSCs [32]. Additionally, many of these studies were conducted in the context of acute infarction, with significant work still needing to be initiated in the setting of HF.

\section{Heart Failure}

Heart failure is a major cause of morbidity and mortality worldwide, with greater than 5.6 million individuals afflicted in the US alone [33]. Heart failure is among the most common diagnoses for hospital admission with estimates of approximately one percent of the western world afflicted and constitutes approximately 400,000 new admissions annually [34]. It is now appreciated that the underlying cellular processes in HF are an interplay of myocardial factors, systemic factors, and local inflammation [35]. Collectively, the disarray of molecular pathways, downstream signaling, and subsequent gene expression culminates in the debilitating clinical disease state of HF. Recent studies have demonstrated that inflammatory cues play a critical role in instigating the onset of HF at the molecular level with cytokines such as interleukin 1 beta (IL-1 $\beta$ ) and nuclear factor kappa B (NF- $\kappa \mathrm{B})$ contributing critically to left ventricular (LV) deterioration [23, 24, 36-39].

\section{Current Treatment of Heart Failure}

Current treatment strategies in HF focus on minimizing disease morbidity, reducing hospitalizations, and prevention of mortality [33]. Despite the significant economic burden as well as morbidity and mortality stemming from HF, no promising treatment modalities to reverse the disease process currently exist. In particular, end-stage HF results in a common final pathway initiated by several signaling mechanisms that are ultimately characterized by myocardial dysfunction and cardiac remodeling. Current treatment options for HF include drug therapy, cardiac resynchronization therapy, mechanical circulatory support, and/or cardiac transplantation [40-43]. While cardiac transplantation does improve mortality and quality of life, it remains a limited therapy given the epidemiologic restriction of donor hearts available. Furthermore, an ever-increasing number of HF patients have no remaining therapies available given these restrictions [44]. Potential new therapies for HF will likely require targeted molecular therapy thus integrating local and systemic inflammation, promoting neoangiogenesis, and developing a methodology by which LV dysfunction can ideally be restored. Cellular therapeutics could allow a greater number of patients afflicted with HF to benefit from therapy than is possible via current advanced heart failure therapies, especially, given consideration that the only true treatment currently available for HF patients remains cardiac transplantation, which is prohibitive given the associated costs and limited donor hearts [7]. Indeed, cell-based therapies and regenerative medicine-directed therapies for HF would significantly change the course of disease progression and patient outcomes. Mesenchymal stem cells represent an ideal candidate in the emerging field of regenerative medicine that could be at the forefront of cell-based therapies for heart failure.

\section{Clinical Trials and MSC-Based Therapies for Heart Failure}

Recently, several clinical trials are ongoing in order to determine the safety and efficacy of MSC-based treatment for acute myocardial infarction (MI), with far fewer trials investigating the use of MSC-based therapies in the setting of HF 
(Table 1) $[45,46]$. Most of the trials utilizing MSCs as a therapeutic option are in ischemic heart failure, and there are little data to date on the treatment in nonischemic cardiomyopathies. Indeed, MSCs have been used in clinical trials to treat both ischemic and nonischemic heart failure with both approaches showing promising results $[47,48]$. While these diseases differ in terms of presence or absence of coronary artery disease, the MSCs are directed at generating new myocardium. One could speculate that MSCs would potentially be more effective in nonischemic disease because the damaged myocardium still has adequate blood supply. In the ischemic HF trials, studies have already started to show improvements in regional and global systolic and diastolic function, reversal of LV remodeling, and enhanced myocardial collateralization and coronary perfusion using the regenerative potential of MSCs [49-53]. Of note, the benefits of MSC therapy appear to be seen in the relative short term, and there remains a question as to the long-term benefits of MSC therapy as in other types of cell-based therapy. This suggests that the paracrine-mediated effects of cell-based therapy may be directly related to cell survival. Mechanistically, much remains to be elucidated as to the exact means by which MSCs achieve reversal of LV remodeling. It has been suggested that limiting inflammation coupled with deposition of extracellular matrix components deposited by MSCs may help limit the total scar size thereby decreasing LV dimensions and possibly improving diastolic function [27, 28, 54-57]. Despite these advances, further investigation into the mechanisms by which MSCs are able to facilitate these actions is warranted. Some studies suggest critical roles played by cytokines, expressed by MSCs or progenitor cells recruited by MSCs, that may be integral to cardiac recovery including insulin like growth factor 1 (IGF-1), hepatocyte growth factor (HGF), and vascular endothelial growth factor (VEGF) [58-62].

With the ever-increasing popularity of MSCs as potential cellular-based therapeutics for HF, a number of clinical trials have recently been completed and several are underway investigating the perspective roles how MSCs could play in the setting of HF events (Table 1). The majority of these trials have looked at MSCs, primarily for the reasons outlined previously $[3,5,6,24,28,30]$. Several trials have attempted to investigate the use of MSCs in the setting of acute and chronic HF, with a variety of strategies on cell delivery. Initial trials focused on events closer to the time of the infarct, with cell administrations occurring in the setting of acute myocardial infarction, with MSCs being investigated in an attempt to prevent ischemic cardiomyopathy [50]. Still, other studies have looked at the therapeutic potential of MSCs in the setting of nonischemic cardiomyopathies including the setting of chemotherapy and dilated cardiomyopathies [47, 63]. However, given the healthcare burden manifested by ischemic chronic HF, later trials have looked at the treatment potential of MSCs outside the time of acute MI [64]. The addition of studies investigating the population of patients afflicted with HF remains paramount given that the disease state remains the leading reason for morbidity and mortality. Furthermore, trials investigating nonischemic HF are needed as well. On a physiologic and biochemical level, chronic ischemic HF changes the microenvironment and biochemical milieu of signaling that occurs, thus altering cardiovascular physiology $[32,65,66]$. In addition to MSCs, other cellbased therapies are also under consideration in clinical trials as potential alternatives for cell-based therapies; however, the majority of trials remain focused on MSCs given their desirable characteristics, ease of use, and accessibility [42, 67-69]. Indeed, no other cell-based therapy for HF continues to hold as much potential as MSCs for a true "off the shelf" approach that can be utilized in autologous or allogeneic modalities.

\section{Results of Clinical Trials with MSCs}

Predominantly, clinical trials have demonstrated that MSCs are safe for administration without increased risk for adverse events [18, 42-44]. Furthermore, results of the previous trials suggest that treatment with MSCs does not increase risk of posttreatment arrhythmias [42]. Additionally, studies have shown significant improvement in patient exercise tolerance $[42,44]$. Investigations have also looked to see if MSCs utilized as concurrent or adjuvant therapies with existing treatment modalities for HF such as in the setting of mechanical circulatory support devices can provide benefits [70]. While many of these clinical trials have found trends towards improvement in New York Heart Association (NYHA) class, the results have not always been statistically significant or with dramatic improvements in treatment versus nontreatment groups. However, this trend seems to be changing. Recent trials have started to demonstrate findings that are statistically significant, likely due to several factors, among which is increasing sample size $[42,44]$. Another factor playing a role in observed results likely lies with MSCs themselves. The route of administration has been a focal point of investigation of MSCs in HF. Initial studies were in the setting of acute myocardial infarction, and intracoronary delivery was the standard practice [71]. The limitations to delivering MSCs by the intracoronary route is the observation that the cells are rapidly washed out. Investigators have tried to increase the dwelling time in the coronary artery by delivering the cells and then occluding the coronary artery. To date, it is not clear that this approach increases the number of cells remaining in the heart. Attempts have been made to investigate alternative approaches as well. Intravenous allogeneic MSCs have been tested in a small pilot study in patients with nonischemic cardiomyopathy [72]. The authors speculate that the anti-inflammatory effects of the MSCs may be the mechanism of action because the cells were delivered intravenously. Other studies have investigated the role of endomyocardial delivery of MSCs and coronary sinus approaches as well, albeit the latter was with bone marrow aspirate and not purely MSCs [32, 47, 73, 74]. However, the route of administration remains an area that requires more investigation.

Studies have quelled previous concerns regarding safety in administration of MSCs [7, 64, 65, 69]. This has even been investigated in the setting of dilated cardiomyopathies [47]. The POSEIDON-DCM trial is a randomized comparison of allogeneic versus autologous MSCs for nonischemic dilated cardiomyopathy delivered transendocardially ([32]). Although in a small trial, the early results are encouraging 


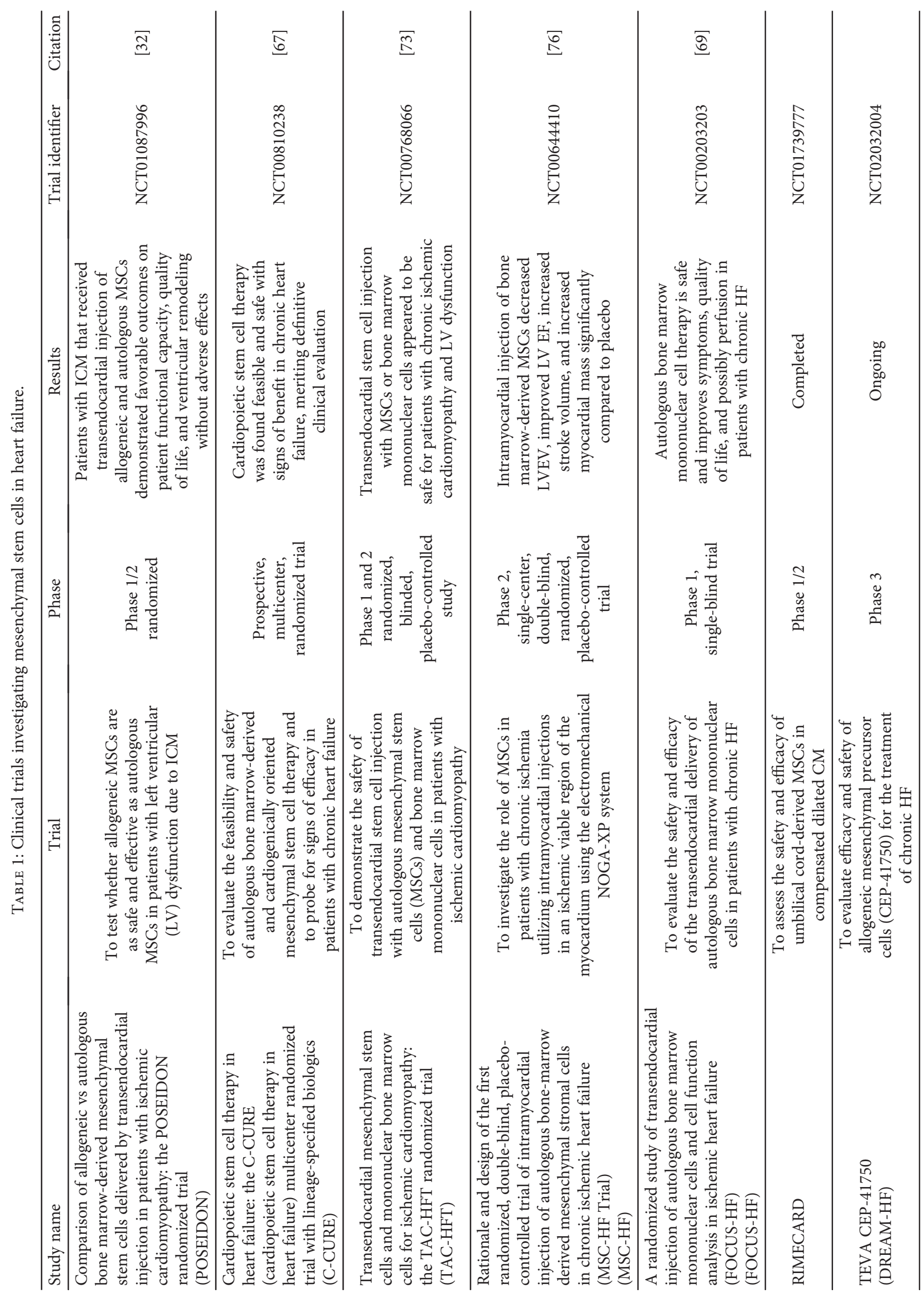




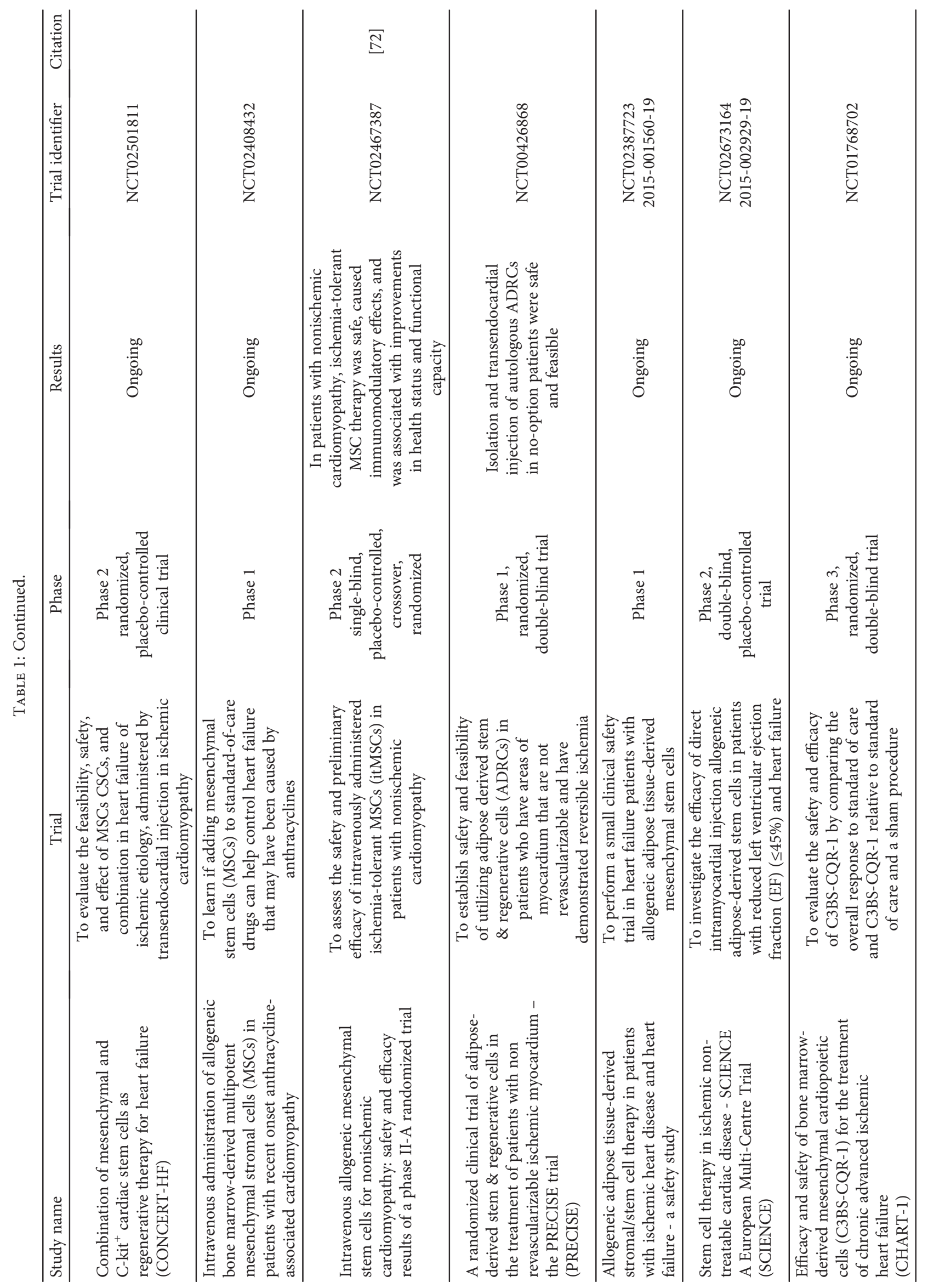


showing that the allogeneic cells increased ejection fraction and functional activity with no significant serious adverse events. The CONCERT-HF trial is an ongoing study investigating the combination of MSCs and c-kit ${ }^{+}$cardiac stem cells in ischemic cardiomyopathy (NCT02501811), and is, interestingly, a trial that employs autologous bone marrowderived MSCs. While the original approach for cell-based therapy in heart failure focused on using autologous cells, investigators are now using allogeneic cells because of the potential to deliver larger numbers of cells without harvesting from the patient. Allogeneic cells can be obtained from younger subjects where the cells may have more regenerative capacity based on protein expression and bioinformatics [3]. Furthermore, it has been suggested that $\mathrm{HF}$, at its central process, is an inflammatory process [36]. In addition, it has been shown that MSCs are potently antiinflammatory [5]. Knowing this, it follows that MSCs could slow and potentially reverse the ill effects of HF, if not too far progressed. Lastly, using allogeneic cells would be less costly and be able to deliver a true "off the shelf" approach. Allogeneic MSC therapy has been shown to be as safe and efficacious as autologous MSC therapy [32, 47, 49, 56, 69, 73, 75-78].

The primary mechanism by which MSCs are able to promote endogenous repair appears to be via paracrine signaling; however, the cells still need to hone to the area of interest and be retained, at least for some duration, to exert their therapeutic effects. Furthermore, the area(s) where MSCs hone to must have at least some vascularization given that MSCs act via paracrine signaling. Current administration of MSCs remains entrenched in traditional approaches including intravenous (IV) and intracardiac (IC) modes of delivery [34, 42-44, 79]. Recent trials have investigated the use of allogeneic MSCs, which has been safe and effective in a comparable capacity to autologous MSC therapeutic strategies [42]. Furthermore, preclinical studies as well as data from clinical trials have suggested that young donor MSCs have different signaling pathways activated when compared with older donor MSCs, given the changing cellular dynamics of aged MSCs [3, 44]. Thus, use of allogeneic young donor MSCs for treatment in an "off the shelf" therapeutic option make MSCs even more favorable as a cell-based therapy modality. This approach is being used in the Dream-HF trial with allogeneic mesenchymal precursor cells (NCT02032004). Indeed, an innovative delivery of MSCs could provide the missing component to propel MSCs as the long-sought-after treatment option for HF.

\section{Delivery of MSCs with Biomaterials}

Delivery with tissue-engineered biomaterials could provide an innovative delivery system to enable MSCs to further develop as a treatment option for HF and other cardiovascular disorders. Administration of MSCs via a venous approach has the risk of cells honing to an area of damage that is outside the heart. While IC administration of MSCs will confirm that MSCs will be present in the heart, often the cells are injected into the scar, which given its lack of adequate vascular supply, would not be hospitable to MSCs as therapeutics.
Biomaterials are gaining increasing interest, especially with the advent of new technologies that allow for innovative treatment modalities. Administration of MSCs with the aid of biomaterials such as a scaffold could potentially resolve some of the perceived issues with MSC delivery in the setting of HF. Mesenchymal stem cells have been used in combination with biomaterials in preclinical studies with some promising results. Investigators injected a self-setting salinized hydroxypropyl methylcellulose seeded with MSCs and showed improvements in LV remodeling and infarct expansion in a rat model of myocardial infarction [80]. Another approach to improve retention of transplanted cells in the diseased heart is to inject the cells in an in situ cross-linked alginate hydrogel [81]. Adipose-derived MSCs embedded in alginate retain their viability, maintain their paracrine potential, and are not immunogenic suggesting that using alginate hydrogels may be a method to enhance delivery of MSCs in the clinical arena. A similar approach encapsulating MSCs in an alginate hydrogel patch has shown potential clinical benefit in a rat infarct model with evidence of improved cardiac function, decreased scar size, and increased peri-infarct vasculature [82].

The ideal scaffold would allow delivery of MSCs in such a way that would allow the cells to exert their paracrine signaling and not to impede the release of these secreted factors. Furthermore, the scaffold used for cell delivery would create not only a vector for delivery of the cells but also a more hospitable microenvironment from which MSCs could direct endogenous repair. It would also be advantageous if the scaffold could help generate its own new blood supply. This would overcome the current dilemmas of how to target MSCs to specific areas of the heart as well as concerns of engraftment in a potentially hostile environment, the postinfarction myocardium. Additionally, a biodegradable scaffold would exist only transiently; once the cells have established, the scaffold would no longer be needed. Among the current hypotheses of MSCs used as therapeutics, one suggested that MSCs typically initiate and direct the early phases of endogenous repair, and once the process is sufficiently underway, these cells are not required and are not retained for longer periods. Ideally, the scaffold would result in minimal inflammation, remain only transiently, not require removal, and promote angiogenesis.

\section{Cellular Scaffolds}

Several approaches have been suggested or are under investigation as cellular delivery alternatives to IV or IC administration of cells. Among these methods are cell- or tissue-based scaffold, electroshock-assisted cell delivery, and polymers for transport [83-86]. Criticisms of cell-based therapies point towards poor retention of cells, insufficient number of cells utilized for therapy, poor engraftment in the hostile conditions of HF, disruption of molecular honing signals, and excess extracellular matrix secondary to fibrosis as the pitfalls of cell administration-based therapies as options for HF. The potential pitfalls described in cell-based therapies can all be overcome by the use of cellular/tissue scaffolds. Previously, we have described a cellular scaffold that has been developed 


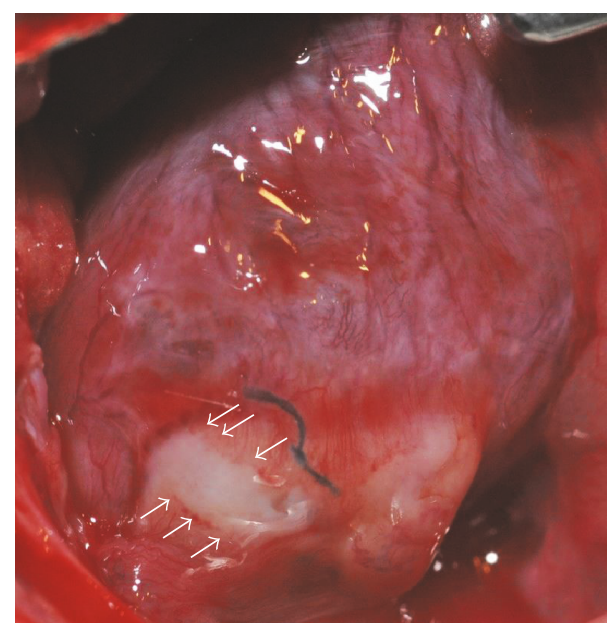

Figure 2: Cellular scaffold (arrows) placed over a scar in a rat heart in vivo 3 weeks after infarction. Three weeks after patch implantation, 6 weeks after infraction, evidence of angiogenesis from the patch to native myocardium was observed.

and demonstrated hemodynamic improvement as well as promotion of angiogenesis (Figure 2) [87, 88]. Such a cellular scaffold would not only allow for adequate delivery in regard to cell number but also location as it could be placed over the region of the scar. Furthermore, the scaffold would provide a more hospitable setting than the surrounding infarct, which would otherwise be much more hostile for MSC function. The scaffold would thus work in conjunction with MSCs to modulate the cellular microenvironment to make it more favorable and promote cellular repairs. MSCs administered with the scaffold would provide the necessary components for increased angiogenesis to occur, while reducing the need for honing and engraftment of MSCs. Utilizing a cellular scaffold, with known cardiovascular improvements in the setting of HF, with MSCs, given their potential as cellular therapeutics, could provide the elusive component necessary to progress cell-based therapies in HF.

\section{Conclusion}

MSCs were once touted as the ideal candidate for cell- and gene-based therapies. They were identified as the cells that would change regenerative medicine via their ability to differentiate into end-cell lines, allowing the shortage of donor organs to become a nonfactor in treatment of many endstage disease states. As MSCs have been further investigated, it is their paracrine signaling that has come to the forefront and become the characteristic that makes them ideal candidates as cellular therapeutics. The ability to modulate the cellular microenvironment through expression of various cytokines and regulation of signal transduction pathways to direct and promote endogenous cellular repair is considered the hallmark function of MSCs. Furthermore, MSCs via their paracrine signaling are able to recruit dormant progenitor cells to aid in the regenerative process. Clinical trials have demonstrated that MSCs are safe and can play as a mainstay of treatment of HF. Novel delivery of MSCs as therapeutics in HF can overcome many of the current pitfalls such as hostile environment of $\mathrm{HF}$ for regenerative medicine and retention of cells. Cellular scaffolds in particular can assure that critical numbers of MSCs are able to reach the target area, whereby MSCs can then direct endogenous cellular repair.

\section{Current and Future Perspectives}

MSCs have been of interests for their potential as cellular therapeutics since their first description 37 years ago. Their ability to differentiate into end-cell lineages enticed investigators to believe that the dream of creating organs in the laboratory had become a reality. It was not until recently that it was determined that the therapeutic potential of MSCs was primarily in their mechanism of paracrine signaling, and not with the differentiation potentials. MSCs have been investigated in a myriad of disease states. Among the most devastating and costly disease of which currently remains is chronic HF. The scope of MSCs as potential therapies in $\mathrm{HF}$ is still in the very early stages. While significant progress is currently being made with ischemic HF clinical trials revealing that MSCs are not only safe for administration but may also provide the much anticipated therapeutic benefit. However, future research is needed to elucidate the ideal delivery of MSCs in the setting of ischemic cardiomyopathies, and research is needed in nonischemic cardiomyopathies as well. The mechanisms at play by which MSCs function to improve molecular and clinical state of HF need to be identified. Furthermore, clinically relevant endpoints of MSC therapy such as exercise time and functional capacity are important metrics to assess as we strive to improve the quality of life in patients with heart failure.

\section{Conflicts of Interest}

The authors have nothing to disclose. The authors declare that there is no conflict of interest regarding the publication of this article.

\section{Acknowledgments}

The authors would like to thank Dr. Joseph Bahl for his help in reviewing the manuscript and the thoughtful discussions in the process of this work. They would also like to acknowledge Taylor Nordan for his assistance in the preparation of this manuscript. This work was supported by the Graduate Medical Education Resident Excellence and Leadership Scholarship (ACP), the Sarver Heart Center, University of Arizona, the WARMER Foundation, and the Martin and Carol Reid Family Charitable Remainder Trust.

\section{References}

[1] P. A. Heidenreich, N. M. Albert, L. A. Allen et al., "Forecasting the impact of heart failure in the United States: a policy statement from the American Heart Association," Circulation Heart Failure, vol. 6, pp. 606-619, 2013.

[2] A. H. Maass, K. Vernooy, S. C. Wijers et al., "Refining success of cardiac resynchronization therapy using a simple score predicting the amount of reverse ventricular remodelling: 
results from the markers and response to CRT (MARC) study," Europace, 2017.

[3] A. C. Pandey, J. A. Semon, D. Kaushal et al., "MicroRNA profiling reveals age-dependent differential expression of nuclear factor $\kappa \mathrm{B}$ and mitogen-activated protein kinase in adipose and bone marrow-derived human mesenchymal stem cells," Stem Cell Research \& Therapy, vol. 2, no. 6, p. 49, 2011.

[4] D. J. Prockop, "Repair of tissues by adult stem/progenitor cells (MSCs): controversies, myths, and changing paradigms," Molecular Therapy, vol. 17, no. 6, pp. 939-946, 2009.

[5] L. A. Ortiz, M. Dutreil, C. Fattman et al., "Interleukin 1 receptor antagonist mediates the antiinflammatory and antifibrotic effect of mesenchymal stem cells during lung injury," Proceedings of the National Academy of Sciences of the United States of America, vol. 104, no. 26, pp. 11002-11007, 2007.

[6] M. P. De Miguel, S. Fuentes-Julian, A. Blazquez-Martinez et al., "Immunosuppressive properties of mesenchymal stem cells: advances and applications," Current Molecular Medicine, vol. 12, no. 5, pp. 574-591, 2012.

[7] J. S. Da Silva and J. M. Hare, "Cell-based therapies for myocardial repair: emerging role for bone marrow-derived mesenchymal stem cells (MSCs) in the treatment of the chronically injured heart," Methods in Molecular Biology, vol. 1037, pp. 145-163, 2013.

[8] E. Ratcliffe, R. J. Thomas, and D. J. Williams, "Current understanding and challenges in bioprocessing of stem cell-based therapies for regenerative medicine," British Medical Bulletin, vol. 100, pp. 137-155, 2011.

[9] A. J. Friedenstein, J. F. Gorskaja, and N. N. Kulagina, "Fibroblast precursors in normal and irradiated mouse hematopoietic organs," Experimental Hematology, vol. 4, no. 5, pp. 267-274, 1976.

[10] C. De Bari, F. Dell'Accio, P. Tylzanowski, and F. P. Luyten, "Multipotent mesenchymal stem cells from adult human synovial membrane," Arthritis and Rheumatism, vol. 44, no. 8, pp. 1928-1942, 2001.

[11] A. Erices, P. Conget, and J. J. Minguell, "Mesenchymal progenitor cells in human umbilical cord blood," British Journal of Haematology, vol. 109, no. 1, pp. 235-242, 2000.

[12] M. J. Hoogduijn, M. J. Crop, A. M. Peeters et al., "Human heart, spleen, and perirenal fat-derived mesenchymal stem cells have immunomodulatory capacities," Stem Cells and Development, vol. 16, no. 4, pp. 597-604, 2007.

[13] P. S. In't Anker, S. A. Scherjon, C. Kleijburg-van der Keur et al., "Amniotic fluid as a novel source of mesenchymal stem cells for therapeutic transplantation," Blood, vol. 102, no. 4, pp. 1548-1549, 2003.

[14] S. Kadiyala, R. G. Young, M. A. Thiede, and S. P. Bruder, "Culture expanded canine mesenchymal stem cells possess osteochondrogenic potential in vivo and in vitro," Cell Transplantation, vol. 6, no. 2, pp. 125-134, 1997.

[15] P. A. Zuk, M. Zhu, P. Ashjian et al., "Human adipose tissue is a source of multipotent stem cells," Molecular Biology of the Cell, vol. 13, no. 12, pp. 4279-4295, 2002.

[16] D. G. Phinney and D. J. Prockop, "Concise review: mesenchymal stem/multipotent stromal cells: the state of transdifferentiation and modes of tissue repair-current views," Stem Cells, vol. 25, no. 11, pp. 2896-2902, 2007.

[17] C. Sanina and J. M. Hare, "Mesenchymal stem cells as a biological drug for heart disease: where are we with cardiac cell-based therapy?," Circulation Research, vol. 117, no. 3, pp. 229-233, 2015.

[18] W. T. Tse, J. D. Pendleton, W. M. Beyer, M. C. Egalka, and E. C. Guinan, "Suppression of allogeneic T-cell proliferation by human marrow stromal cells: implications in transplantation," Transplantation, vol. 75, no. 3, pp. 389-397, 2003.

[19] S. C. Hung, R. R. Pochampally, S. C. Chen, S. C. Hsu, and D. J. Prockop, "Angiogenic effects of human multipotent stromal cell conditioned medium activate the PI3K-Akt pathway in hypoxic endothelial cells to inhibit apoptosis, increase survival, and stimulate angiogenesis," Stem Cells, vol. 25, no. 9, pp. 2363-2370, 2007.

[20] J. K. Biehl and B. Russell, "Introduction to stem cell therapy," The Journal of Cardiovascular Nursing, vol. 24, no. 2, pp. 98 103, 2009, quiz 104-105.

[21] D. J. Prockop, “"Stemness" does not explain the repair of many tissues by mesenchymal stem/multipotent stromal cells (MSCs)," Clinical Pharmacology and Therapeutics, vol. 82, no. 3, pp. 241-243, 2007.

[22] K. G. Shyu, B. W. Wang, H. F. Hung, C. C. Chang, and D. T. Shih, "Mesenchymal stem cells are superior to angiogenic growth factor genes for improving myocardial performance in the mouse model of acute myocardial infarction," Journal of Biomedical Science, vol. 13, no. 1, pp. 47-58, 2006.

[23] M. Bujak and N. G. Frangogiannis, "The role of IL-1 in the pathogenesis of heart disease," Archivum Immunologiae et Therapiae Experimentalis (Warsz), vol. 57, no. 3, pp. 165176, 2009.

[24] B. W. Van Tassell, R. A. Arena, S. Toldo et al., "Enhanced interleukin-1 activity contributes to exercise intolerance in patients with systolic heart failure," PLoS One, vol. 7, no. 3, article e33438, 2012.

[25] K. S. Telukuntla, V. Y. Suncion, I. H. Schulman, and J. M. Hare, "The advancing field of cell-based therapy: insights and lessons from clinical trials," Journal of the American Heart Association, vol. 2, no. 5, article e000338, 2013.

[26] P. R. Vulliet, M. Greeley, S. M. Halloran, K. A. MacDonald, and M. D. Kittleson, "Intra-coronary arterial injection of mesenchymal stromal cells and microinfarction in dogs," Lancet, vol. 363, no. 9411, pp. 783-784, 2004.

[27] R. H. Lee, A. A. Pulin, M. J. Seo et al., "Intravenous hMSCs improve myocardial infarction in mice because cells embolized in lung are activated to secrete the anti-inflammatory protein TSG-6," Cell Stem Cell, vol. 5, no. 1, pp. 54-63, 2009.

[28] L. Li, S. Zhang, Y. Zhang, B. Yu, Y. Xu, and Z. Guan, "Paracrine action mediate the antifibrotic effect of transplanted mesenchymal stem cells in a rat model of global heart failure," Molecular Biology Reports, vol. 36, no. 4, pp. 725-731, 2009.

[29] T. Freyman, G. Polin, H. Osman et al., "A quantitative, randomized study evaluating three methods of mesenchymal stem cell delivery following myocardial infarction," European Heart Journal, vol. 27, no. 9, pp. 1114-1122, 2006.

[30] J. D. Richardson, A. G. Bertaso, P. J. Psaltis et al., "Impact of timing and dose of mesenchymal stromal cell therapy in a preclinical model of acute myocardial infarction," Journal of Cardiac Failure, vol. 19, no. 5, pp. 342-353, 2013.

[31] K. E. Hatzistergos, H. Quevedo, B. N. Oskouei et al., "Bone marrow mesenchymal stem cells stimulate cardiac stem cell proliferation and differentiation," Circulation Research, vol. 107, no. 7, pp. 913-922, 2010. 
[32] J. M. Hare, J. E. Fishman, G. Gerstenblith et al., "Comparison of allogeneic vs autologous bone marrow-derived mesenchymal stem cells delivered by transendocardial injection in patients with ischemic cardiomyopathy: the POSEIDON randomized trial," The Journal of the American Medical Association, vol. 308, no. 22, pp. 2369-2379, 2012.

[33] V. L. Roger, A. S. Go, D. M. Lloyd-Jones et al., "Heart disease and stroke statistics-2012 update: a report from the American Heart Association," Circulation, vol. 125, no. 1, pp. e2-e220, 2012.

[34] L. W. Miller and E. D. Missov, "Epidemiology of heart failure," Cardiology Clinics, vol. 19, no. 4, pp. 547-555, 2001.

[35] A. M. Katz, "The "modern" view of heart failure: how did we get here?," Circulation: Heart Failure, vol. 1, no. 1, pp. 63-71, 2008.

[36] A. Abbate, B. W. Van Tassell, I. M. Seropian et al., "Interleukin-1 $\beta$ modulation using a genetically engineered antibody prevents adverse cardiac remodelling following acute myocardial infarction in the mouse," European Journal of Heart Failure, vol. 12, no. 4, pp. 319-322, 2010.

[37] M. Bujak, M. Dobaczewski, K. Chatila et al., "Interleukin-1 receptor type I signaling critically regulates infarct healing and cardiac remodeling," The American Journal of Pathology, vol. 173, no. 1, pp. 57-67, 2008.

[38] C. Freund, R. Schmidt-Ullrich, A. Baurand et al., "Requirement of nuclear factor- $\kappa \mathrm{B}$ in angiotensin II- and isoproterenol-induced cardiac hypertrophy in vivo," Circulation, vol. 111, no. 18, pp. 2319-2325, 2005.

[39] J. Heineke and J. D. Molkentin, "Regulation of cardiac hypertrophy by intracellular signalling pathways," Nature Reviews Molecular Cell Biology, vol. 7, no. 8, pp. 589-600, 2006.

[40] M. Attisani, P. Centofanti, M. La Torre et al., "Advanced heart failure in critical patients (INTERMACS 1 and 2 levels): ventricular assist devices or emergency transplantation?," Interactive Cardiovascular and Thoracic Surgery, vol. 15, no. 4, pp. 678-684, 2012.

[41] N. Garg, A. Senthilkumar, M. B. Nusair, N. Goyal, R. K. Garg, and M. A. Alpert, "Heart failure with a normal left ventricular ejection fraction: epidemiology, pathophysiology, diagnosis and management," The American Journal of the Medical Sciences, vol. 346, no. 2, pp. 129-136, 2013.

[42] F. Kamdar, R. John, P. Eckman, M. Colvin-Adams, S. J. Shumway, and K. Liao, "Postcardiac transplant survival in the current era in patients receiving continuous-flow left ventricular assist devices," The Journal of Thoracic and Cardiovascular Surgery, vol. 145, no. 2, pp. 575-581, 2013.

[43] F. H. Verbrugge, P. De Vusser, M. Rivero-Ayerza et al., "Cardiac resynchronization therapy with or without defibrillator: experience from a high-volume Belgian implantation centre," Acta Cardiologica, vol. 68, no. 1, pp. 37-45, 2013.

[44] M. J. Lenzen, E. Boersma, W. J. Reimer et al., "Under-utilization of evidence-based drug treatment in patients with heart failure is only partially explained by dissimilarity to patients enrolled in landmark trials: a report from the Euro Heart Survey on Heart Failure," European Heart Journal, vol. 26, no. 24, pp. 2706-2713, 2005.

[45] J. M. Hare, "Translational development of mesenchymal stem cell therapy for cardiovascular diseases," Texas Heart Institute Journal, vol. 36, no. 2, pp. 145-147, 2009.

[46] B. Trachtenberg, D. L. Velazquez, A. R. Williams et al., "Rationale and design of the transendocardial injection of autologous human cells (bone marrow or mesenchymal) in chronic ischemic left ventricular dysfunction and heart failure secondary to myocardial infarction (TAC-HFT) trial: a randomized, double-blind, placebo-controlled study of safety and efficacy," American Heart Journal, vol. 161, no. 3, pp. 487-493, 2011.

[47] J. M. Hare, D. L. DiFede, A. C. Rieger et al., "Randomized comparison of allogeneic versus autologous mesenchymal stem cells for nonischemic dilated cardiomyopathy: POSEIDONDCM trial," Journal of the American College of Cardiology, vol. 69, no. 5, pp. 526-537, 2017.

[48] A. N. Patel, T. D. Henry, A. A. Quyyumi et al., "Ixmyelocel$\mathrm{T}$ for patients with ischaemic heart failure: a prospective randomised double-blind trial," Lancet, vol. 387, no. 10036, pp. 2412-2421, 2016.

[49] L. C. Amado, A. P. Saliaris, K. H. Schuleri et al., "Cardiac repair with intramyocardial injection of allogeneic mesenchymal stem cells after myocardial infarction," Proceedings of the National Academy of Sciences of the United States of America, vol. 102, no. 32, pp. 11474-11479, 2005.

[50] J. M. Hare, J. H. Traverse, T. D. Henry et al., “A randomized, double-blind, placebo-controlled, dose-escalation study of intravenous adult human mesenchymal stem cells (prochymal) after acute myocardial infarction," Journal of the American College of Cardiology, vol. 54, no. 24, pp. 2277-2286, 2009.

[51] H. C. Quevedo, K. E. Hatzistergos, B. N. Oskouei et al., "Allogeneic mesenchymal stem cells restore cardiac function in chronic ischemic cardiomyopathy via trilineage differentiating capacity," Proceedings of the National Academy of Sciences of the United States of America, vol. 106, no. 33, pp. 14022-14027, 2009.

[52] K. H. Schuleri, L. C. Amado, A. J. Boyle et al., "Early improvement in cardiac tissue perfusion due to mesenchymal stem cells," American Journal of Physiology - Heart and Circulatory Physiology, vol. 294, no. 5, pp. H2002-H2011, 2008.

[53] G. V. Silva, S. Litovsky, J. A. Assad et al., "Mesenchymal stem cells differentiate into an endothelial phenotype, enhance vascular density, and improve heart function in a canine chronic ischemia model," Circulation, vol. 111, no. 2, pp. 150-156, 2005.

[54] J. A. Dixon, R. C. Gorman, R. E. Stroud et al., "Mesenchymal cell transplantation and myocardial remodeling after myocardial infarction," Circulation, vol. 120, Supplement 11, pp. S220-S229, 2009.

[55] Y. Y. Du, S. H. Zhou, T. Zhou et al., "Immuno-inflammatory regulation effect of mesenchymal stem cell transplantation in a rat model of myocardial infarction," Cytotherapy, vol. 10, no. 5, pp. 469-478, 2008.

[56] S. Ishikane, K. Yamahara, M. Sada et al., "Allogeneic administration of fetal membrane-derived mesenchymal stem cells attenuates acute myocarditis in rats," Journal of Molecular and Cellular Cardiology, vol. 49, no. 5, pp. 753-761, 2010.

[57] C. Schneider, K. Jaquet, S. Geidel et al., "Transplantation of bone marrow-derived stem cells improves myocardial diastolic function: strain rate imaging in a model of hibernating myocardium," Journal of the American Society of Echocardiography, vol. 22, no. 10, pp. 1180-1189, 2009.

[58] A. I. Caplan and J. E. Dennis, "Mesenchymal stem cells as trophic mediators," Journal of Cellular Biochemistry, vol. 98, no. 5, pp. 1076-1084, 2006.

[59] T. Nakamura, S. Mizuno, K. Matsumoto, Y. Sawa, H. Matsuda, and T. Nakamura, "Myocardial protection from ischemia/ reperfusion injury by endogenous and exogenous HGF," The 
Journal of Clinical Investigation, vol. 106, no. 12, pp. 15111519, 2000.

[60] D. M. Pedrotty and L. E. Niklason, "Angiogenesis therapies for cardiovascular disease," Current Opinion in Anaesthesiology, vol. 16, no. 1, pp. 3-9, 2003.

[61] S. Sadat, S. Gehmert, Y. H. Song et al., "The cardioprotective effect of mesenchymal stem cells is mediated by IGF-I and VEGF," Biochemical and Biophysical Research Communications, vol. 363, no. 3, pp. 674-679, 2007.

[62] K. Urbanek, M. Rota, S. Cascapera et al., "Cardiac stem cells possess growth factor-receptor systems that after activation regenerate the infarcted myocardium, improving ventricular function and long-term survival," Circulation Research, vol. 97, no. 7, pp. 663-673, 2005.

[63] F. Ezquer, J. Gutierrez, M. Ezquer, C. Caglevic, H. C. Salgado, and S. D. Calligaris, "Mesenchymal stem cell therapy for doxorubicin cardiomyopathy: hopes and fears," Stem Cell Research \& Therapy, vol. 6, p. 116, 2015.

[64] V. Jeevanantham, M. Butler, A. Saad, A. Abdel-Latif, E. K. Zuba-Surma, and B. Dawn, "Adult bone marrow cell therapy improves survival and induces long-term improvement in cardiac parameters: a systematic review and meta-analysis," Circulation, vol. 126, no. 5, pp. 551-568, 2012.

[65] S. Chen, Z. Liu, N. Tian et al., "Intracoronary transplantation of autologous bone marrow mesenchymal stem cells for ischemic cardiomyopathy due to isolated chronic occluded left anterior descending artery," The Journal of Invasive Cardiology, vol. 18, no. 11, pp. 552-556, 2006.

[66] A. B. Mathiasen, M. Haack-Sorensen, and J. Kastrup, "Mesenchymal stromal cells for cardiovascular repair: current status and future challenges," Future Cardiology, vol. 5, no. 6, pp. 605-617, 2009.

[67] J. Bartunek, A. Behfar, D. Dolatabadi et al., "Cardiopoietic stem cell therapy in heart failure: the C-CURE (cardiopoietic stem cell therapy in heart failure) multicenter randomized trial with lineage-specified biologics," Journal of the American College of Cardiology, vol. 61, no. 23, pp. 2329-2338, 2013.

[68] A. B. Mathiasen, E. Jorgensen, A. A. Qayyum, M. HaackSorensen, A. Ekblond, and J. Kastrup, "Rationale and design of the first randomized, double-blind, placebo-controlled trial of intramyocardial injection of autologous bonemarrow derived mesenchymal stromal cells in chronic ischemic heart failure (MSC-HF trial)," American Heart Journal, vol. 164, no. 3, pp. 285-291, 2012.

[69] E. C. Perin, G. V. Silva, T. D. Henry et al., "A randomized study of transendocardial injection of autologous bone marrow mononuclear cells and cell function analysis in ischemic heart failure (FOCUS-HF)," American Heart Journal, vol. 161, no. 6, pp. 1078-1087.e3, 2011.

[70] D. D. Ascheim, A. C. Gelijns, D. Goldstein et al., "Mesenchymal precursor cells as adjunctive therapy in recipients of contemporary left ventricular assist devices," Circulation, vol. 129, no. 22, pp. 2287-2296, 2014.

[71] S. Li, X. Wang, J. Li et al., "Advances in the treatment of ischemic diseases by mesenchymal stem cells," Stem Cells International, vol. 2016, Article ID 5896061, 11 pages, 2016.

[72] J. Butler, S. E. Epstein, S. J. Greene et al., "Intravenous allogeneic mesenchymal stem cells for nonischemic cardiomyopathy: safety and efficacy results of a phase II-A randomized trial," Circulation Research, vol. 120, no. 2, pp. 332-340, 2017.
[73] A. W. Heldman, D. L. DiFede, J. E. Fishman et al., "Transendocardial mesenchymal stem cells and mononuclear bone marrow cells for ischemic cardiomyopathy: the TAC-HFT randomized trial," The Journal of the American Medical Association, vol. 311, no. 1, pp. 62-73, 2014.

[74] A. N. Patel, S. Mittal, G. Turan et al., "REVIVE trial: retrograde delivery of autologous bone marrow in patients with heart failure," Stem Cells Translational Medicine, vol. 4, no. 9, pp. 1021-1027, 2015.

[75] S. L. Chen, W. W. Fang, F. Ye et al., "Effect on left ventricular function of intracoronary transplantation of autologous bone marrow mesenchymal stem cell in patients with acute myocardial infarction," The American Journal of Cardiology, vol. 94, no. 1, pp. 92-95, 2004.

[76] A. B. Mathiasen, A. A. Qayyum, E. Jorgensen et al., "Bone marrow-derived mesenchymal stromal cell treatment in patients with severe ischaemic heart failure: a randomized placebo-controlled trial (MSC-HF trial)," European Heart Journal, vol. 36, no. 27, pp. 1744-1753, 2015.

[77] E. C. Perin, J. T. Willerson, C. J. Pepine et al., "Effect of transendocardial delivery of autologous bone marrow mononuclear cells on functional capacity, left ventricular function, and perfusion in chronic heart failure: the FOCUS-CCTRN trial," The Journal of the American Medical Association, vol. 307, no. 16, pp. 1717-1726, 2012.

[78] K. H. Schuleri, G. S. Feigenbaum, M. Centola et al., “Autologous mesenchymal stem cells produce reverse remodelling in chronic ischaemic cardiomyopathy," European Heart Journal, vol. 30, no. 22, pp. 2722-2732, 2009.

[79] X. Wang, M. N. Jameel, Q. Li et al., "Stem cells for myocardial repair with use of a transarterial catheter," Circulation, vol. 120, Supplement 11, pp. S238-S246, 2009.

[80] E. Mathieu, G. Lamirault, C. Toquet et al., "Intramyocardial delivery of mesenchymal stem cell-seeded hydrogel preserves cardiac function and attenuates ventricular remodeling after myocardial infarction," PLoS One, vol. 7, no. 12, article e51991, 2012.

[81] B. Follin, M. Juhl, S. Cohen et al., "Human adipose-derived stromal cells in a clinically applicable injectable alginate hydrogel: phenotypic and immunomodulatory evaluation," Cytotherapy, vol. 17, no. 8, pp. 1104-1118, 2015.

[82] R. D. Levit, N. Landazuri, E. A. Phelps et al., "Cellular encapsulation enhances cardiac repair," Journal of the American Heart Association, vol. 2, no. 5, article e000367, 2013.

[83] B. Assmus, U. Fischer-Rasokat, J. Honold et al., "Transcoronary transplantation of functionally competent BMCs is associated with a decrease in natriuretic peptide serum levels and improved survival of patients with chronic postinfarction heart failure: results of the TOPCARE-CHD registry," Circulation Research, vol. 100, no. 8, pp. 1234-1241, 2007.

[84] B. Assmus, D. H. Walter, F. H. Seeger et al., "Effect of shock wave-facilitated intracoronary cell therapy on LVEF in patients with chronic heart failure: the CELLWAVE randomized clinical trial," The Journal of the American Medical Association, vol. 309, no. 15, pp. 1622-1631, 2013.

[85] E. A. Bocchi, F. Bacal, G. Guimaraes et al., "Granulocytecolony stimulating factor or granulocyte-colony stimulating factor associated to stem cell intracoronary infusion effects in non ischemic refractory heart failure," International Journal of Cardiology, vol. 138, no. 1, pp. 94-97, 2010.

[86] L. A. Sanchez, C. E. Guerrero-Beltran, A. M. Cordero-Reyes, G. Garcia-Rivas, and G. Torre-Amione, "Use of stem cells in 
heart failure treatment: where we stand and where we are going," Methodist DeBakey Cardiovascular Journal, vol. 9, no. 4, pp. 195-200, 2013.

[87] J. Lancaster, E. Juneman, T. Hagerty et al., "Viable fibroblast matrix patch induces angiogenesis and increases myocardial blood flow in heart failure after myocardial infarction," Tissue Engineering, Part A, vol. 16, no. 10, pp. 3065-3073, 2010.

[88] H. M. Thai, E. Juneman, J. Lancaster et al., "Implantation of a three-dimensional fibroblast matrix improves left ventricular function and blood flow after acute myocardial infarction," Cell Transplantation, vol. 18, no. 3, pp. 283-295, 2009. 

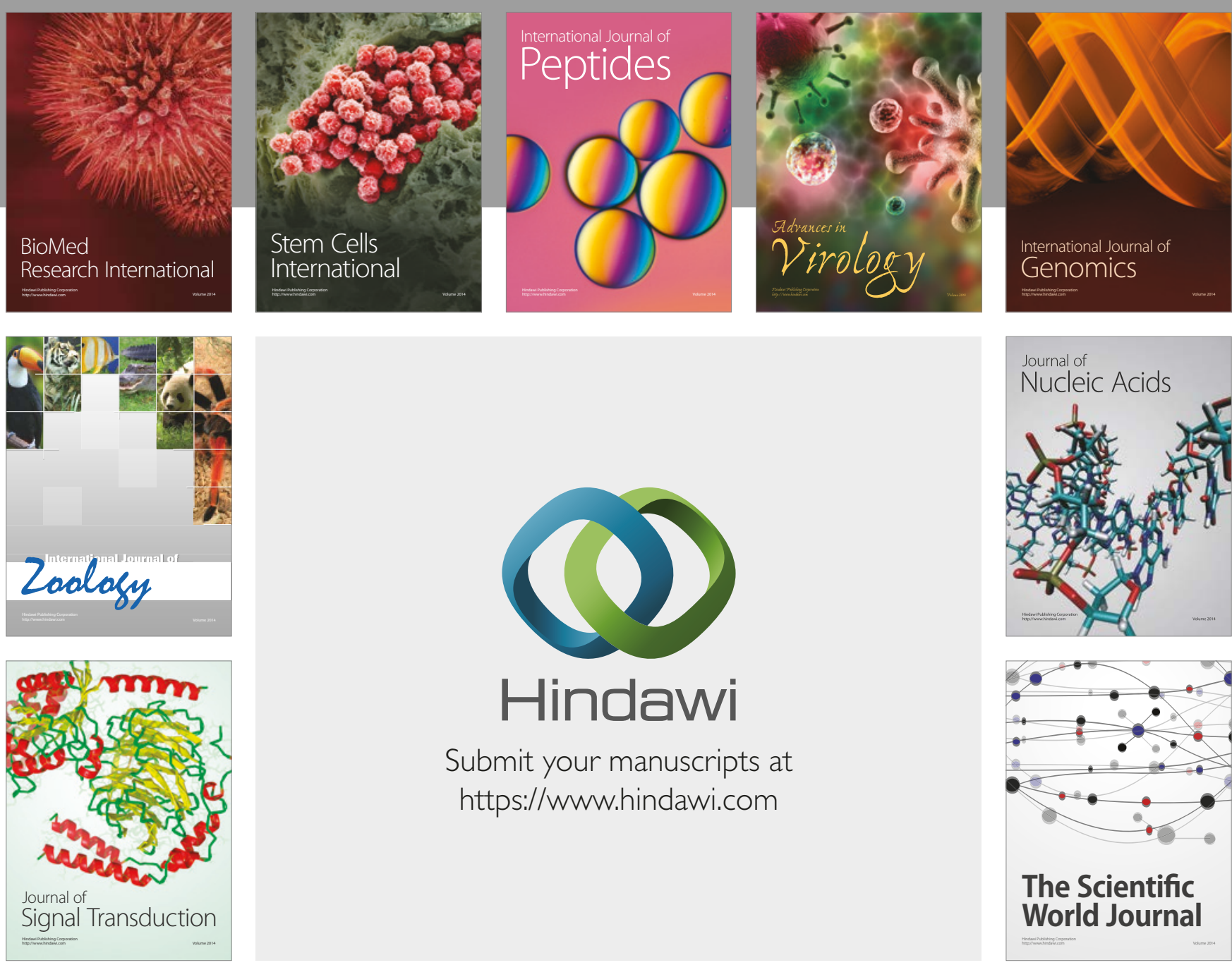

Submit your manuscripts at

https://www.hindawi.com
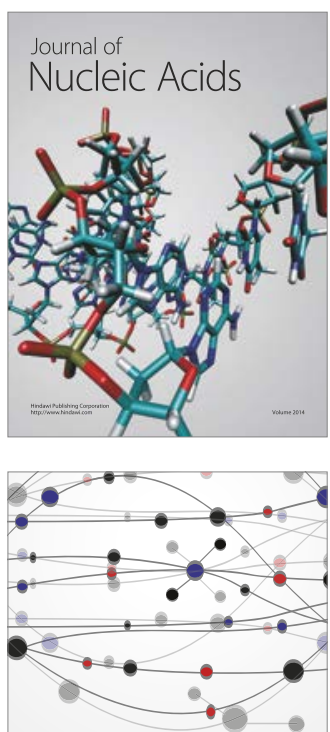

The Scientific World Journal

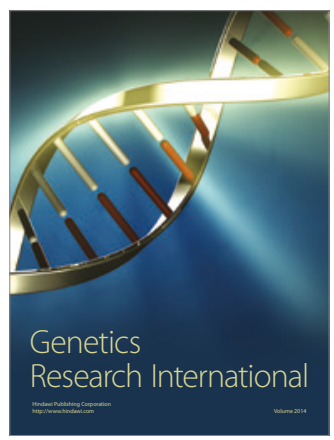

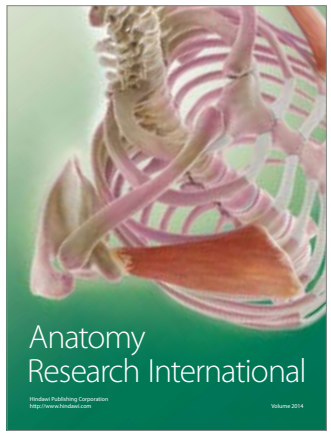

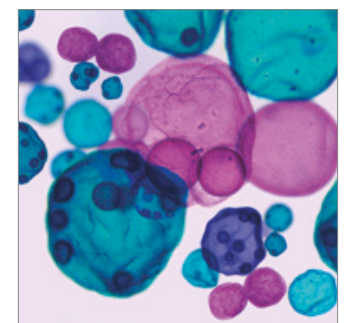

International Journal of Microbiology
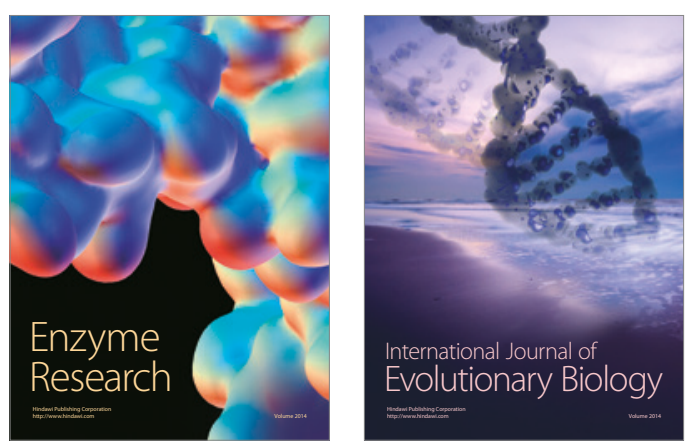
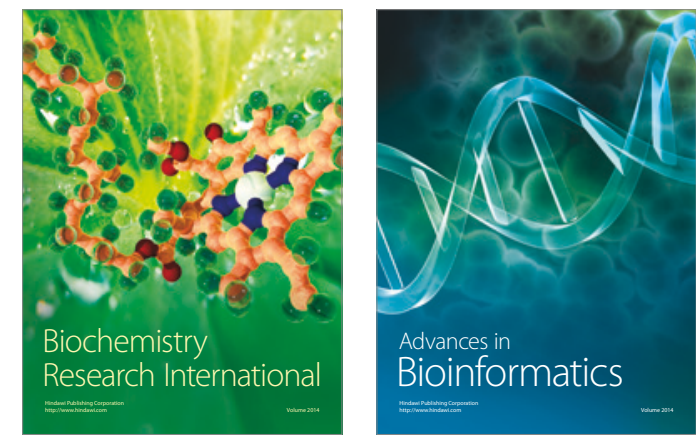

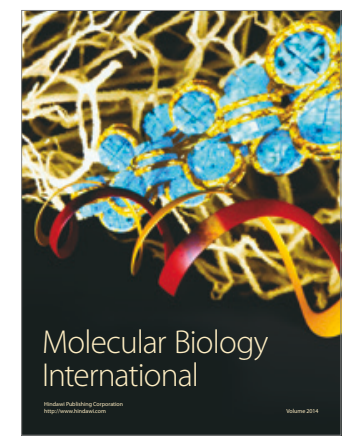

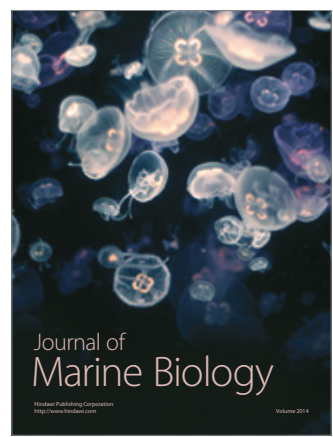

